\title{
An Energy Efficient Cluster Selection Optimization using Evolutionary Imperialist Competitive Algorithm
}

\author{
Chaitra H.V. \\ Nitte Institute of Technology \\ Assistant Professor \\ Department of CSE
}

\author{
Ravikumar G.K., PhD \\ Research Guide \\ CSE BOARD VTU, \\ Belgaum
}

\begin{abstract}
Wireless Communications is one of the fastest growing segments in the communications industry. Wireless Network is the network that facilitates communication among two or more devices connected through the standard network protocols, without network cabling. Due to the battery constrained the network performance will get reduced i.e. If the energy of the wireless sensor node (WSN's) is drained, recharging of the sensor nodes in unattended environment is very difficult. As WSN nodes are usually battery-powered devices, the important and most critical aspects to face concern is how to reduce the energy consumption of WSN nodes, so that the network lifetime can be enhanced to an extent. Routing the Data in sensor nodes plays a vital role in transferring the data to the base station (BS). Different types of routing algorithm have been used such multihopping, grid based, hierarchical based and clustering based such LEACH, HEED etc... In this we have focused on incorporating clustering technique based on evolutionary technique namely ICA cluster optimization to improve the lifetime of the sensor nodes. We compare our proposed clustering model with LEACH protocol and analyze its efficiency.
\end{abstract}

\section{General Terms}

Imperialist competitive algorithm cluster optimization (ICACO), challenges , clustering technique

\section{Keywords}

WSN, Clustering, data aggregation, Evolutionary technique.

\section{INTRODUCTION}

In wireless sensor network communications have enabled low cost, low-power, multifunctional sensor nodes for the development that are small size and short distances communication. The sensor networks use tiny (small) sensor nodes, which consist of sensing, data processing, and collaborating (communication) component. A sensor node is a node in a wireless sensor network which is capable of performing rarefaction, sensory information for congregation and communicating with other consecutive nodes in the sensor network. We live in a world filled with sensors. The buildings that we work in, have sensors monitoring temperature, occupancy, smoke and fire, and security. Our cars contain dozens if not hundreds of sensors, monitoring engine performance, braking, and passenger safety equipment and so on. Manufacturing environments need sensors because you cannot control what you cannot measure. Making products, while meeting safety, quality and efficiency targets, requires a lot of sensors. Sensors have become much smaller, less expensive and lower power in the last few decades.

A Wireless sensor network (WSN) has important application such as remote tracking ecological monitoring, detecting wildfire or signs of any catastrophic phenomena, intruder detection in military applications. This has been enabled by the availability, especially in recent years, of sensors that are small in size. These nodes are furnished with wireless interface (WI) with which they can connect with each other to form a network. Wireless sensor networks (WSN's) consist of sensor node with transmission and communication capabilities. As wireless sensor node is usually batterypowered devices, the critical and important aspects that face concern are how to reduce the power consumption of sensor nodes, so that the network energy lifetime can be enhanced to an extent.

\section{THE MAJOR CHALLENGES IN WIRELESS SENSOR NETWORKS}

1. Communications: Large data transfer is simply not feasible in wireless sensor network (WSNs), which unlike other wireless hardware (laptop NICs, home-routers, etc.), WSN communication protocols are designed to meet lowpower specifications. This is partially due to the fact that sensor nodes do not have access to a constant source of electricity (to recharge batteries), therefore the transmission bandwidth must be one that is efficient and yet supports balanced data-rates.

2. Networking: WSNs which consist of large number of sensor nodes often lead to unpredictable results and frequent topological changes. These dynamic alterations cause an imbalance of energy consumption, higher collision rates, and channel contention among sensor devices.

Multi-hopping and clustering techniques have been known to reduce the number of simultaneous one-to-one connections between sensor nodes and base stations; however, these techniques alone are not enough to guarantee data integrity and protection against loss of information.

3. Management: WSNs require adept management protocol techniques in order to utilize their true potential in terms of suitability, usability and functionality in real applications. Firstly, power management is very important given that sensor nodes have limited access to energy resources such as batteries. Secondly, node failure management must be addressed appropriately given that WSNs are prone to hardware failures caused by noise interference, node device failures, and buggy software. Finally, the management of components and subsystems such as communication modules, sensors, and periphery units, must be addressed carefully given that improper use can lead to irregular node behaviour and an increase of data loss.

4. Data Aggregation: In wireless sensor network (WSN) data processing requires development of new secure and energy efficient technique enabling fusion of relatively large amount of data. Data aggregation has to be done over a massively Distributed database with constraint resources (battery). How can we be sure that the final, high level sensing results delivered by the system is an accurate reflection of the state of 
the environment, when the sensors are deployed, where there is insufficient energy and bandwidth to record all the raw data?

\section{CLUSTERING TECHNIQUE AND ITS EFFECTS ON LIFETIME OF SENSOR NETWORK}

Clustering has been well received as an effective way to reduce the energy consumption of a wireless sensor network. Clustering is defined as the process of selecting a set of wireless sensor nodes to be cluster heads for a given wireless sensor network. Therefore, data traffic generated at each sensor node can be sent via cluster heads to the sink/base station. Clustering is also used for data aggregation, where the cluster heads aggregate the data collected at the cluster members. Hierarchical routing protocols proved to have sufficient reduction in energy consumption of the wireless sensor network (WSN). In hierarchical routing protocols, tree is created with numbers of clusters and a head node is assigned to each cluster. Head nodes are the leaders of their groups. They have some responsibilities like collection and aggregation the data from cluster node of their corresponding clusters heads and transmitting the aggregated data to the base station (BS). This aggregated data in the head nodes which reduces energy consumption in the network by reducing the information to be sent to the BS which result in less energy consumption and increases the network life time. Here we focus on LEACH routing protocol and analyse its benefits and the drawback issue it consists. Due to the optimization problem in selecting cluster head of leach and to improve lifetime here we propose an efficient cluster optimization technique based on evolutionary computational technique namely Imperialist Competitive algorithm cluster optimization (ICACO) algorithm.

\section{LEACH}

Low-Energy Adaptive Clustering Hierarchy (namely LEACH) is proposed by Heinzlman et al., is the first approach developed using hierarchical clustering approach in wireless sensor network(WSN's). In LEACH protocol, the wireless sensor nodes of the network will be organizing and form themselves into local clusters, with one of the nodes of them behaving as the cluster head/leader ( $\mathrm{CH})$ [5].The basic operation of LEACH protocol is carried out into multiple rounds, where each round is divided into two phases,

\section{(A) Set-up phase and}

\section{(B) Steady-state phase.}

In phase one each node considers itself as $\mathrm{CH}$ (cluster head) for the current round but it is decides based on the predefined percentage of $\mathrm{CHs}$ and how many times the node has been a $\mathrm{CH}$ in previous rounds. The judgement/decision is taken by the node choosing a random value between 0 to 1 . The node will consider as a $\mathrm{CH}$ if the value is less than the given threshold value for current round:

Benefit / Advantages of LEACH protocol:

$>$ The node that select as a $\mathrm{CH}$ in a particular round will not be selected as the cluster head $(\mathrm{CH})$ in next round, so all node can equally share the load in the network.

$>$ The TDMA avoids unnecessary collision of cluster heads $(\mathrm{CHs})$ in wireless sensor network (WSN).
Drawback / Disadvantages of LEACH protocol:

$>$ It sends data directly from cluster heads $(\mathrm{CHs})$ to the base station (BS) without intermediate node which utilizes more energy for transmission.

$>$ Because of probabilistic approach of cluster head $(\mathrm{CH})$ selection, $\mathrm{CH}$ may select having low energy in it.

\section{PROPOSED IMPERIALIST COMPETITIVE ALGORITHM CLUSTER OPTIMIZATION (ICACO)}

We propose a new and efficient cluster model based on evolutionary computation algorithm which divides the cluster into sub clusters by using ICACO (Imperialist Competitive Algorithm cluster optimization) and form a sub cluster to select the cluster head.

Imperialism is the procedure of spreading the control of an imperial beyond its own limits in a cluster. An imperialist manage the wireless sensor network (WSN's) by direct rule or by less control of arcade to divided cluster using sufficient energy. Our ICACO algorithm is used to find the sufficient energy of wireless sensor network to detect in efficient way for using this approach. To divide the cluster into sub cluster by using Imperialist Competitive Algorithm (ICA) is one of the most powerful algorithms; it has been used extensively to solve different kinds of optimization problems.

Each group of the sensor node is called a sub cluster; some of the sub cluster in the nodes is selected to be the imperialist. The sensor node has encountered the selected neighboring to the task, the sensor node has better range and algorithm has efficiently utilized the clusters. All the networks of initial sensor node are divided among the imperialists based on their function for instance and choosing cluster head having sufficient energy remaining sub cluster nodes are cluster child. The network in each of domain starts moving towards their relevant position and changes the place in the new one. The control of each sub cluster is made up of imperialist require function and networks. It is based on cluster control. The cluster which is weaker (less energy) than the others, loses its networks until there will be no network in that. This action is extinction of the weakest (least energy) cluster, its imperialist is considered as the finest Network. The level of imperialist Challenges is when there is only one domain is the optimum point. ICACO can provide more accurate/precise solutions in less computational time/cost when compared to other algorithm and improves the cluster energy.

Imperialist Competitive Algorithm (ICA) is a challenging and new evolutionary optimization method which is inspired by imperialistic competition [6-8]. Like any other evolutionary algorithms, it also starts with an initial population which is here called country and it is divided into two types of imperialists and colonies which together form an empires. Imperialistic competition (IC) among these empires forms the proposed evolutionary algorithm namely ICACO. During this competition, weak empires collapse and powerful ones take it's possession of their colonies. Imperialistic competition converges to a state in which there exists only one empire and colonies have the same cost functions value as the imperialist.

This algorithm (ICACO) starts by generating a set of initial candidate random solution in search space of the OP (optimization problem). The generated RP(random points) are known as the initial countries. Here in our algorithm Countries are particle in Particle Swarm Optimization (PSO) 
and Chromosome in Genetic Algorithm (GA) and it is a collection of values of a contender solution of OP (optimization problem). The CF (cost function) of the $\mathrm{OF}$ (optimization factor) determines the power of each country. Based on these factor(power), some of the best initial countries (i.e. the countries with the least(best) cost function (CF) value), become the Imperialists and start taking control of other colonies (are called countries) and form the initial empire [9].

The two main mechanism of this algorithm are Assimilation and Revolution. Revolution brings sudden random changes in the position of some of the countries in the search space. Assimilation makes the colonies of each empire get closer/nearer to the imperialist state in the space of sociopolitical characteristics (cluster optimization search space). During revolution and assimilation a colony might reach (get closer) a better position and has the chance/better factor to take the control of the entire empire and interchange/replace the current imperialist state of the empire [10]. Imperialistic Competition is another part of this algorithm. All the empires try to win this war and take possession of colonies of other empires. In each step of the algorithm, based on their energy/power, all the empires have a chance to take control of one or more of the colonies of the weakest empire [9].

The pseudo code of our proposed Imperialist competitive algorithm cluster optimization (ICACO) is as follows:

1) First select some initial random points on the function and initialize the empires.

2) Then Assimilation -Move the colonies toward their relevant imperialist

3) Revolution -Randomly change the position of some colonies.

4) If there is a colony in an empire which has lower cost than the imperialist, exchange the positions of that imperialist and the Colony.

5) Unite the similar empires.

6) Compute the total cost of all empires.

7) Pick the weakest (fragile) colony (colonies) from the weakest empires and give it to one of the empires (Imperialistic competition)

8) Eliminate the powerless empires.

9) If stop conditions is satisfied, stop, if not go to 2 .

Our proposed ICACO technique takes various parameters into consideration to enhance the Sensor network lifetime. These parameters are residual energy in the cluster head, required energy to send a message/packet toward the sink node and atleast required energy for receiving a k-bit packet/message by the cluster head and send it to sink/base station. In order to evaluate our algorithm, we simulated our protocol in sensoria simulator and compared it with LEACH protocol.

\section{RESULT}

The system environment used is windows 7 enterprises 64-bit operating system. We have used sensoria simulator which is based on C\# programming and used dot net framework 4.0 visual studios 2010 . We have conducted simulation sturdy on following parameter for network lifetime and throughput efficiency and compared our proposed ICACO with LEACH protocol.

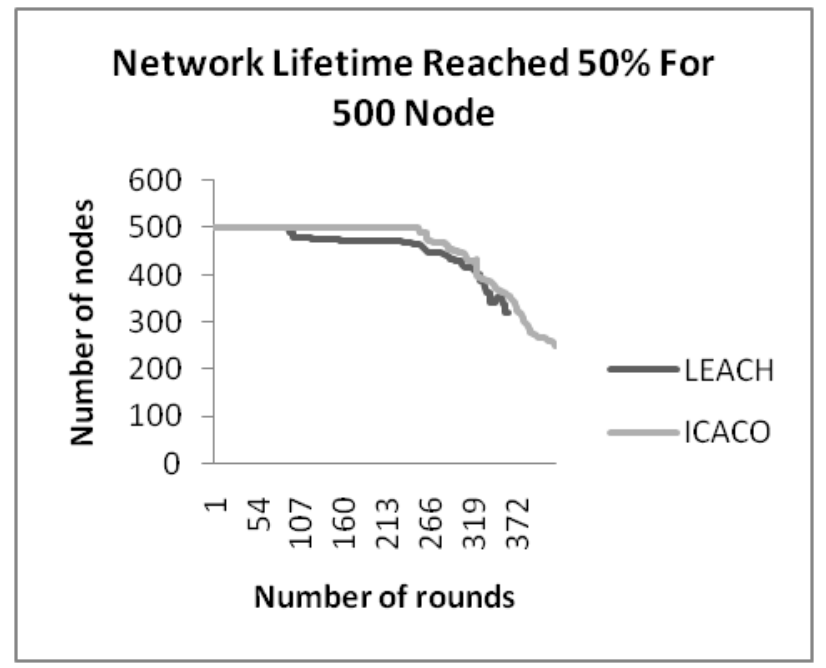

Figure 1: Network lifetime $\mathbf{5 0 \%}$ graph for 500 nodes.

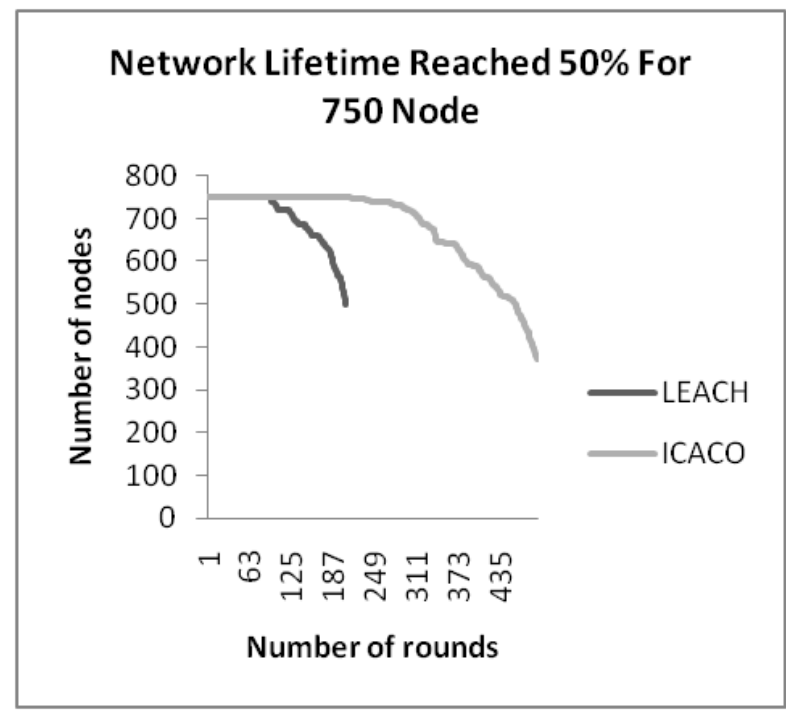

Figure 2: Network lifetime $50 \%$ graph for 750 nodes.

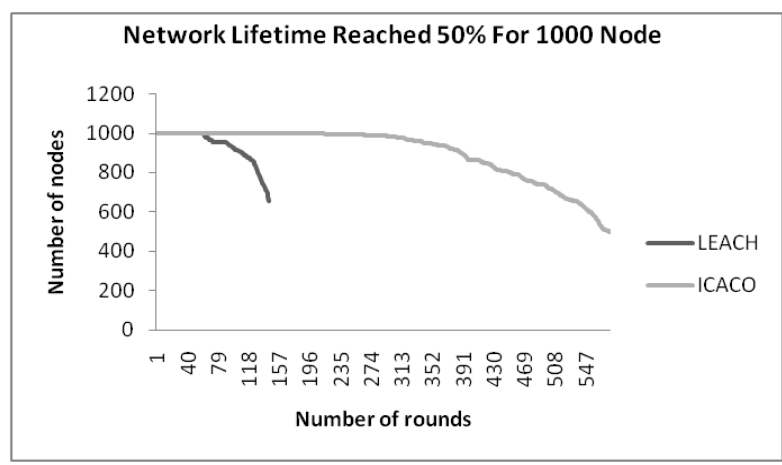

Figure 3: Network lifetime 50\% graph for 1000 nodes.

Network life time comparison of LEACH vs ICACO: From figure 1, 2 and 3 we can see that the proposed ICACO ICA based clustering perform better than LEACH in term lifetime efficiency. The experimental result shows that the energy efficiency of LEACH was stable when sensor node number is 
equal to 500 but when the node increased to 750 and 1000 the life time performance of LEACH degrades significantly. The proposed ICACO improves the lifetime of network by over $14 \%, 58 \%$ and $74 \%$ approximately when sensor node equal to 500,750 and 1000 respectively.

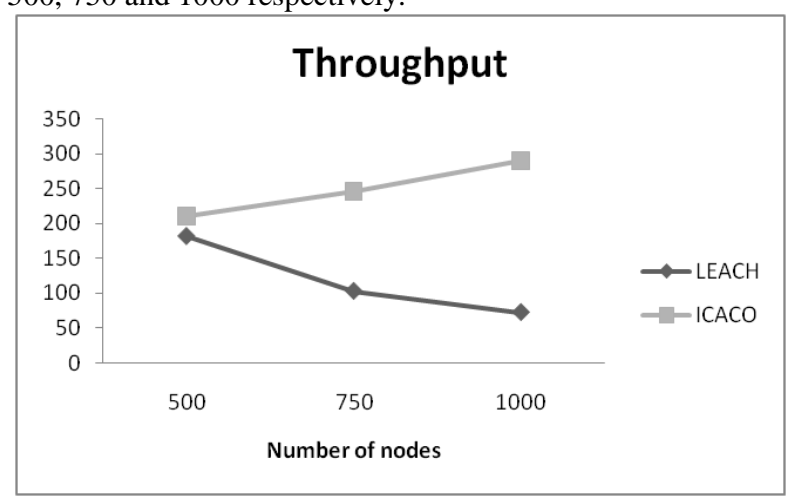

Figure 4: Network Throughput

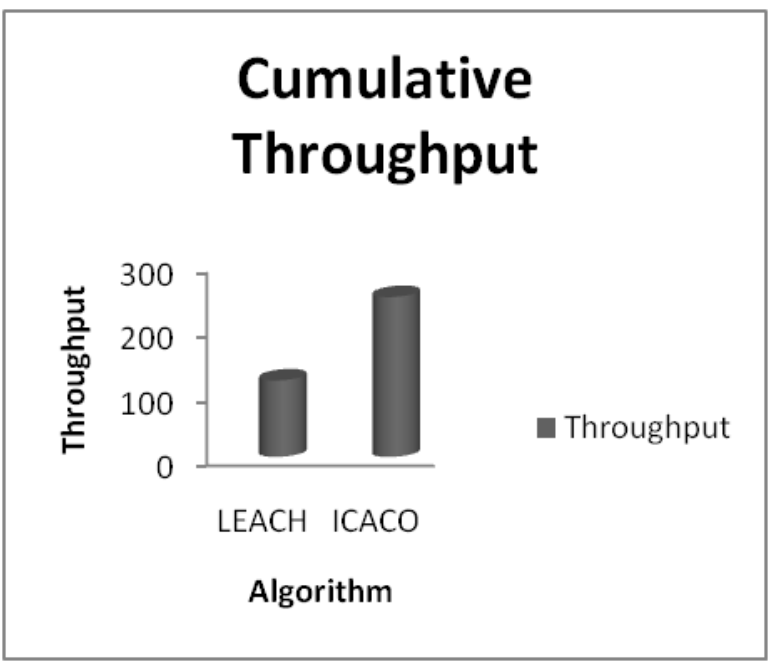

Figure: 5 Cumulative Throughput

Network throughput comparison of LEACH vs ICACO: From figure 4 we can see that the proposed ICACO clustering perform better than LEACH in term of network throughput efficiency. The experimental result shows that the throughput of LEACH decreases when we increases the number of node to 500, 750 and 1000 and the network throughput performance our proposed ICACO algorithm gets better when we increased node to $500,750,1000$. The proposed ICACO improves the throughput of network by over $13 \%, 59 \%$ and $75 \%$ approximately when sensor node equal to 500,750 and 1000 respectively. From figure 5 we can see that the proposed ICACO clustering perform better than LEACH in term of cumulative network throughput efficiency. We have taken the cumulative throughput by varying the node size to 500,750 and 1000 and found it improves the throughput performance by $52 \%$ over LEACH.

\section{CONCLUSION}

The existing clustering protocol (LEACH) is not efficient and suffers in term of life time of network so there was a need new clustering protocol to increase network lifetime. Here we proposed an ICA based clustering protocol to improve energy efficiency in sensor network. Our proposed ICACO algorithm improved lifetime of network by over $14 \%, 58 \%$ and $74 \%$ approximately over LEACH when sensor node equal to 500, 750 and 1000 respectively and the overall network throughput performance by $52 \%$ over LEACH. Experimental results show that the proposed ICA performs better than LEACH in term lifetime efficiency and network throughput. In future work we would conduct simulation to check the performance of other network parameter such as node decay rate, packet delay and by varying node and check how the proposed protocol perform by varying the simulation area size and changing the position of base station to the middle of sensor network area.

\section{REFERENCES}

[1] M. Welsh and G. Mainland, "Programming Sensor Networks Using Abstract Regions," in Proc. USENIX NSDI Conf., Mar. 2004.

[2] A. Boulis, C. Han, and M. B. Srivastava, "Design and Implementation of a Framework for Efficient and Programmable Sensor Networks," in Proc. ACM MobiSys Conf., May 2003.

[3] Younis, O.; Fahmy,S HEED: A hybrid, energy-efficient, distributed clustering approach for adhoc sensor networks. IEEE Trans. Mobile Computing. 2004, 3, 366379.

[4] Jung, S.; Han, Y.; Chung, T. The Concentric Clustering Scheme for Efficient Energy Consumption in the PEGASIS In Proceedings of the 9th International Conference on Advanced Communication Technology, Gangwon-Do, Korea, 12-14 February 2010; pp. 260265.

[5] Heinzelman, W.R.; Chandrakasan, A.; Balakrishnan, H.Energy-Efficient Communication Protocol for Wireless Micro sensor Networks. In Proceedings of the 33rd Annual Hawaii International Conference on System Sciences, Maui, HI, USA, 4-7 January 2010; pp. 10-19.

[6] R. Rajabioun, E. Atashpaz, C. Lucas, "Colonial Competitive Algorithm as a Tool for Nash Equilibrium Point Achievement", Lecture Notes In Computer Science;Vol. 5073, Proc. of the Intl. conf. on Computational Science and Its Applications, Part II, pp.680-695, 2008.

[7] B. Oskouyi, E. Atashpaz-Gargari, N. Soltani, C. Lucas, "Application of Imperialist Competitive Algorithm for Materials Property Characterization from Sharp Indentation Test", to be appeared in International Journal of Engineering Simulation, 2009.

[8] A. M. Jasour, E. Atashpaz, C. Lucas, "Vehicle Fuzzy Controller Design Using Imperialist Competitive Algorithm", Second First Iranian Joint Congress on Fuzzy and Intelligent Systems, Tehran, Iran, 2011.

[9] Atashpaz-Gargari, E., Lucas, C., 2012. "Imperialist Competitive Algorithm: An Algorithm for Optimization Inspires by Imperialistic Competition". IEEE Congress on Evolutionary Computation, Singapore.

[10] Nazari-Shirkouhi, S.; Eivazy, H.; Ghodsi, R.; Rezaie, K.; Atashpaz-Gargari, E. (2010) "Solving the Integrated Product Mix-Outsourcing Problem by a Novel MetaHeuristic Algorithm: Imperialist Competitive Algorithm". Expert Systems with Applications 37 (12): $7615-7626$. 
[11] Pooja Singh, Vikas Pareek and Anil K Ahlawat, "Performance Comparison of Energy Efficient Protocols for Wireless Sensor Networks" International Journal of Computer Applications (0975 - 8887) Volume 90 - No 4, March 2014

[12] Yamuna Devi C R, S H Manjula, K R Venugopal, L M Patnaik, "Multi-hop Route Discovery Using Opportunistic Routing for Wireless Sensor Networks" International Journal of Innovative Technology and Exploring Engineering (IJITEE) ISSN: 2278-3075, Volume-3, Issue-12, May 2014
[13] Dr.G.Padmavathi, Mrs.D.Shanmugapriya, 2009, A Survey of Attacks, Security Mechanisms and Challenges in Wireless Sensor Networks, (IJCSIS) International Journal of Computer Science and Information Security.

[14] Mousam Dagar and Shilpa Mahajan, Data Aggregation in Wireless Sensor Network: A Survey, ISSN 0974-2239 Volume 3, Number 3 (2013), pp. 167-174.

[15] Kiran Maraiya, Kamal Kant, Nitin Gupta, Wireless Sensor Network: A Review on Data Aggregation, International Journal of Scientific \& Engineering Research Volume 2, Issue 4, April -2011 1 ISSN 22295518. 